\title{
PRIVACY PRESERVED HEALTHCARE SYSTEM FOR WIRELESS BODY AREA NETWORKS
}

\author{
DAMANPREET KAUR ${ }^{1} \&$ RUCHIKA GUPTA ${ }^{2}$ \\ ${ }^{I}$ Research Scholar, Department of Computer Science Engineering,Chandigarh University, Gharuan (Punjab), India \\ ${ }^{2}$ Associate Professor, Computer Science Engineering, University Institute of Engineering, Chandigarh University, Mohali, \\ (Punjab), India
}

\begin{abstract}
Today's era is camouflaged with various types of technologies and gadgets that are significantly dealing with sports, entertainment, military; where an enormous data is generated through sensors. Wireless body area networks is a technology that aggregates the patient data store into the server via a communication route. To secure the patient's physiological data, identity, and location information. This paper presents a model based on Elliptic Curve Cryptography (ECC) for securing the patient information where the Trusted Third Party (TTP) works on the K-anonymity parameter with K-1 dummy query users to maintain the confidentiality of the data and location privacy of the user. Our result shows that ECC is an encryption technique with a small key size, exhibits fast encryption, and provide more security than RSA. It is efficiently preserving the real-time location of the user. ECC is more scalable and good for hand hold devices with more computing power and better storage than RSA. The experimental results of the proposed scenario show the performance matrix including encryption time, decryption time, message size varies with $K$ - anonymity parameter value.

KEYWORDS: Wireless Body Area Network, Privacy, Security, Location, Trusted Third Party, Cryptography
\end{abstract}

Received: Jun 08, 2020; Accepted: Jun 28, 2020; Published: Sep 11, 2020; Paper Id.: IJMPERDJUN20201166

\section{INTRODUCTION}

Wireless body area network is one of the fastest growing area these days. WBAN sends miniaturized scale sensors inside or on the outside of human bodies to continually screen the imperative indications of patients. Sensors transfer the gathered physiological information to the clinical help desk stage by means of remote medium and specialists then analyze patient detail at regular time interval as shown in Fig. 1. WBAN architecture shown in Fig. 2. is segregated in three tiers. In tier: 1 various sensors collect all the data and store into the sink node. In tier: 2 sink node is connected with a personal server that transfers the data to the local server (or remote base station). In the tier: 3 data are stored into the hospital database and only authorized doctors can view that data. In-body and onbody are two types of sensors are used in the WBAN. Sensors are mounted on the patient's body and gather all the data into the sink node connected with the personal server [1]. Through the network, data is stored into the hospital database, hence based on the body parameters doctor can prescribe the treatment [2]. According to the Health Insurance Portability and Accountability Act (HIPAA) 1996. It has determined a lot of obligatory security rules to ensure the individual health data [3]. For providing the security of data we use Elliptic Curve Cryptography (ECC). 


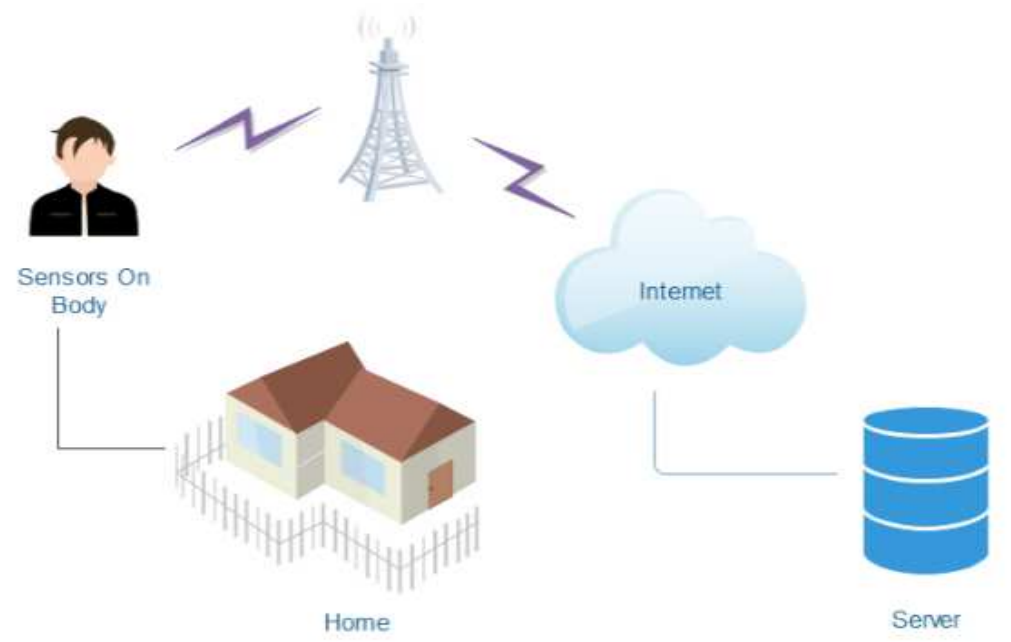

Figure 1: WBAN Architecture.

It is used to preserve the real-time location of the patient. It is generally valuable for versatile applications as it has the capacity to give elevated level security with low computing power and battery asset. ECC is a public key cryptosystem which is utilized to create the public key and the private key so as to scramble and decode the information [4]. A Trusted Third Party plays an important role in this privacy technique to achieve more secure authentication Table: 1 shows ECC is more scalable and good for hand held devices.

The remainder of the paper is organized as follows: Section 2 highlights the literature review and security requirements, section 3 depicts problem formulation, section 4 present proposed model, section 5 exhibits the experimental results, and in the last section shown conclusion of the paper.

\section{Cryptography}

Cryptography is a technique for securing data using the key pair. The message is converted into an encrypted format and at the receiver, decrypts the message by using private key and get the original message. Encryption and decryption are done to maintain the secrecy of the data.

\section{Encryption Techniques}

(a) Symmetric key encryption - In this encryption method sender and receiver, both has the same key pair for encryption and decryption. Examples of symmetric key encryption are blowfish, message digest (MD5), Advanced Encryption Standard (AES), etc. Symmetric key cipher executes in the block cipher or stream cipher.

Stream cipher: In a stream cipher plaintext digits are scrambled each in turn and the change of progressive digits shifts during the encryption state, as the encryption of every digit is subjected to the present state.

Block cipher: In block cipher fixed length of block stores the information. Various operations are performed to make the data more secure.

(b) Public key encryption: It is somewhat similar to the symmetric key encryption [4]. In such a framework, any individual can scramble a message utilizing the recipient's public key, however that scrambled message must be decoded with the recipient's private key. Examples of public key encryption are RSA, ECC, etc. 


\section{Security Requirements in WBAN}

Security and privacy are very significant factors in WBAN. The following security components should be acknowledged.

- Data Integrity: Integrity principles states that the content of the message is not changed after the sender sends it and before the receiver receives it. Only authorized person can access the original message. For example, patient sent its medical information through communication channel and safely received by the authorized doctor without any modification in between.

- Authentication: It is a process of verifying the identity of the user. Patient enter its unique identity and password, if the user identification match with the database then user can check its appointment, previous medical history and other personal information. Doctor can verify the patient with unique identification and prescribe the treatment according to the reports [3].

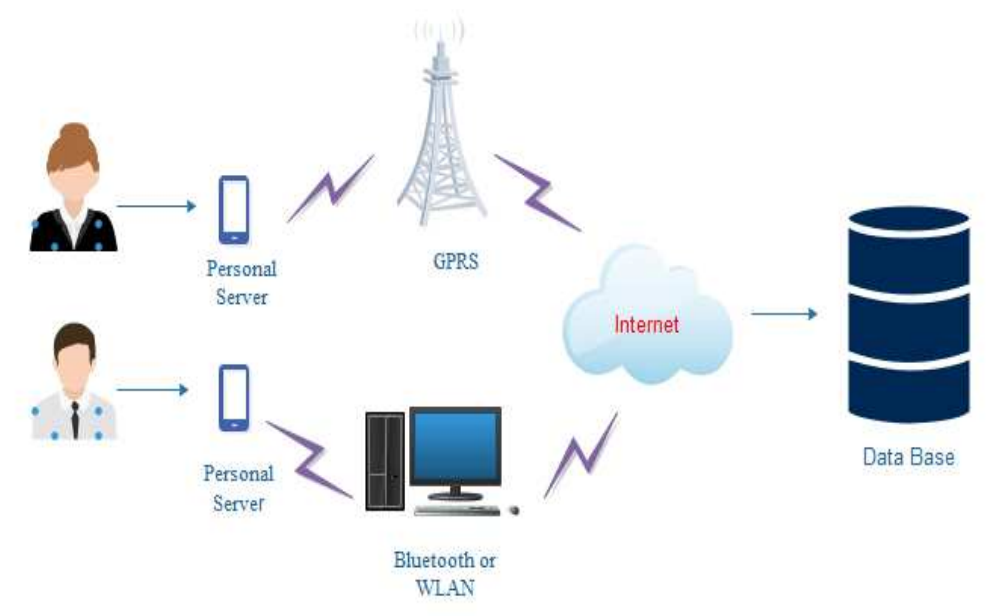

Figure 2: Communication in WBAN.

- Data Confidentiality: It protects sensitive information from unauthorized user, only authorized person can have access to medical records and personal information. For example, the secrecy of patient information should keep secret between the specialist and patient [1].

- Scalability: It means to add several users in the system and properly manage. Some basic requirements have to be fulfilled like authentication, confidentiality, integrity. Several users can connect to hospital's database; more require a database to perform various other tasks like an emergency list of patients and old age people who suffered from the same disease. The workload is divided to easily identify the problem. Maintain the records are much easier. Make a type of system that can be easily managed even if there is any kind of update is required.

- Data Authenticity: Data can be considered authentic; its original form of data does not modify after its creation. Authentic data represent the real-world scenario, originality of data should not be modified if some processing has been done. For example, doctor can send the reports to the patient and ensure that it should not be altered after its generation, the data must be in original form [5].

- Flexibility: The term flexibility refers to easily adapt the changes according to the nature of the network resources, design requirements, topology, etc. If any other type of network connects with our network, it can easily adapt the 
design requirements of our network and communication is effectively done between the nodes. The WBAN network should be flexible so that nodes can easily communicate with the sink node and communication should not be interrupted [6].

- Data Freshness: Data freshness is an essential aspect that supports the integrity and confidentiality of data. Data packets sent over the communication channel, checked whether they are in proper format or not. Freshness format is divided into two types; strong freshness and weak freshness. In the former, we know that packets are sent properly. In the weak freshness, packets latency delay is checked and the record is stored in the database [9].

\section{LITERATURE REVIEW}

In [1], authors discuss WBAN sensors observe the patient's wellbeing exercises. Along these lines, the quantity of sensor hubs sent for WBAN relies upon various elements, for example, physiological parameters. A hub can be any gadget for example, telephone, PC, etc. Information is sent through a communication channel, threads, assaults, and other system issues. These security issues can privacy at stake so It's essential to make sure about the information safely transmitted through transmission channel. Konan et al. [4], propose remote batch authentication scheme between user and application provider. It is a combination of the Elliptic Curve Cryptography and Bilinear Paring Scheme. ECC is a key generating faster, smaller, and efficient cryptosystem keys with low computational cost and device battery usage. In the bilinear pairing two cyclic groups that group is describe a bilinear mapping. In this design a strong mutual batch certificate less authentication scheme between the controller and application provider. Wang et al. [6], propose Privacy-Preserving Priority Classification scheme (PPC) patient's encrypted data is clarified at the WBAN gateway, it classifies all the data from the sender's side and passes the packets according to their priorities and the highest priority medical packet transfer to hospital first. PPC scheme is efficient in computational cost and communication overhead. Zhen et al. [7], propose security assurance procedure empowers dispersed hub verification, information mark and Raft agreement. Specifically, use of blockchain innovation to accomplish dispersed transmission, to guarantee the honesty of information transmission and the anonymity of patient personality protection; joined with MEC can accomplish low latency and high accuracy of delicate information administrations. On the premise, the Merkle tree improvement model can approve the wellspring of private physiological information in WBAN. The algorithm can ensure the uprightness of delicate physiological information. The authors in [8], present a plan to protect area security, termed VIC-PRO, to secure location of the customer close by region ensure utilizing geometrical changes. VIC-PRO algorithm computes the $\mathrm{K}$ clients and performs and changes their position with transformation. Compute the geometric reflection points and add the translation transformation. Query is sent to the LBS provider and after the results, the query sends to the anonymizer. It compares the original position with the fake position, it manages safeguarding region protection alongside securing client identification. The authors in [10], discuss hybrid solution HYB for faster and secure communication. HYB pre-processing the user's location data. Congregation model is used for calculating the centroid of the considerable number of clients included, certain location of user is not revealed. TP (Third Party) has no information about client's genuine area. HYB is a technique to modified the input data and secure the current location of the user. In [11], authors discuss CAST calculation, client communicate with other peers, trust build on the basis of P2P network development. Every single peer makes own small database and store all the information. Instead of central node use the decentralized approach to explore the new group member on the basis of trust. $\mathrm{P} 2 \mathrm{P}$ groups are formed on the basis of similar interests and tendencies. This algorithm provides low latency privacy preserved location. 
The authors in [12], explain exploratory review about the different strategies. Two types of approaches used in this centralized and decentralized. Three types of location-based services pull LBS, Push LBS and tracking LBS. If a silent attacker tracks your activities, it is important to protect the user's identity or personal data. LBS service filtering the immense stuff available on the Internet according to customer's current setting. customers can see basic information that enables them to take instructed decisions on the spot, like picking shopping mall in a particular range. Olakanmi et al. [13], propose a lightweight two-way however perturbation design for obfuscation both the characters and estimations of the sensor in the wearable WBAN framework. Data sent through the source to destination and in between there are some steps like the registration phase and the perturb generation parameters are used. This scheme secured the data and preserving the privacy of WBAN in E-Health. Khan et al. [14], propose multiple biometrics to generate a 160-bit random key for inter sensor communication for two signals ECG and EEG. Compare the similar features and verify message with the Message Authentication Code (MAC). Multiple biometrics security techniques are used in ubiquitous mobile healthcare and remote base station for indoor and outdoor patients for more effective communication. In [15], authors explain the ECC technique. ECC is used to protect the patient's data in WBAN. To avoid the man-in-middle assault, sink node does not hold the identity directly but holds the encrypted data, third party cannot decrypt the data since the attacker does not have the private key of the receiver. The receiver can identify the verification procedure through MAC code the data is sent by the sink node or not. This strategy utilizes the symmetric cipher calculation such as DES and modified feistel algorithm to scramble or decode the patient's clinical information. In [16], authors explain RSA and ECC both are examples, of asymmetric key cryptography RSA is a technique that encrypts the text and length of the text in the hundreds or more than that. To overcome the limitations of RSA, develop more secure encryption technique. ECC it is based on the Elliptic Curve over a finite field. The two points on a curve and add to the third point in it. Generation of key is faster and provide more secure communication than RSA technique.

Table 1: Comparison between RSA and ECC

\begin{tabular}{|l|l|l|}
\hline \multicolumn{1}{|c|}{ Parameters } & \multicolumn{1}{c|}{ ECC } & \multicolumn{1}{c|}{ RSA } \\
\hline Key Sizes & Key pairs are shorter than RSA & Key pairs are larger than ECC \\
\hline Key Generation & Faster speed for generating the key & Slower than ECC \\
\hline Encryption & Faster process than RSA & $\begin{array}{l}\text { Good speed but unfortunately slower } \\
\text { than ECC }\end{array}$ \\
\hline Decryption & Slower than RSA & Faster speed in the decryption process \\
\hline $\begin{array}{l}\text { Computing power and } \\
\text { battery resources }\end{array}$ & $\begin{array}{l}\text { ECC handles the same amount of request in the } \\
75 \text { milliseconds. }\end{array}$ & $\begin{array}{l}\text { RSA certificate handle only the 450 } \\
\text { requests per second }\end{array}$ \\
\hline $\begin{array}{l}\text { Efficiency for Small } \\
\text { Devices }\end{array}$ & It takes less Memory, good for small devices & Less efficient for small devices \\
\hline Scalability & Scalable & Scalability is not optimal \\
\hline Certificates & Use hybrid SSL for ECC & Trusted root certificate \\
\hline
\end{tabular}

Table 2: Bits Comparison between RSA and ECC

\begin{tabular}{|c|c|}
\hline RSA(Bits) & ECC(Bits) \\
\hline 1024 & 160 \\
\hline 2048 & 224 \\
\hline 3072 & 256 \\
\hline 7680 & 384 \\
\hline 15360 & 521 \\
\hline
\end{tabular}




\section{PROBLEM FORMALIZATION}

The earlier technique is used for preserving the privacy of WBAN users with more complexity and larger key size. This new approach is providing the security of real-time location services of a patient and using the ECC to preserve the current location data.

Problem Statement 'A patient is in critical condition; an unauthorized user have access of patient's location coordinates and personal information'.

WBANs empowers the remote checking and constant administration of medicinal service data. This effort will target the scenario a patient's emergency (heart attack, caught fire). The patient sends the query 'Q' to the server. ECC technique for encryption and decryption with $\mathrm{K}$ - anonymity parameter to maintain the confidentiality of the patient data.

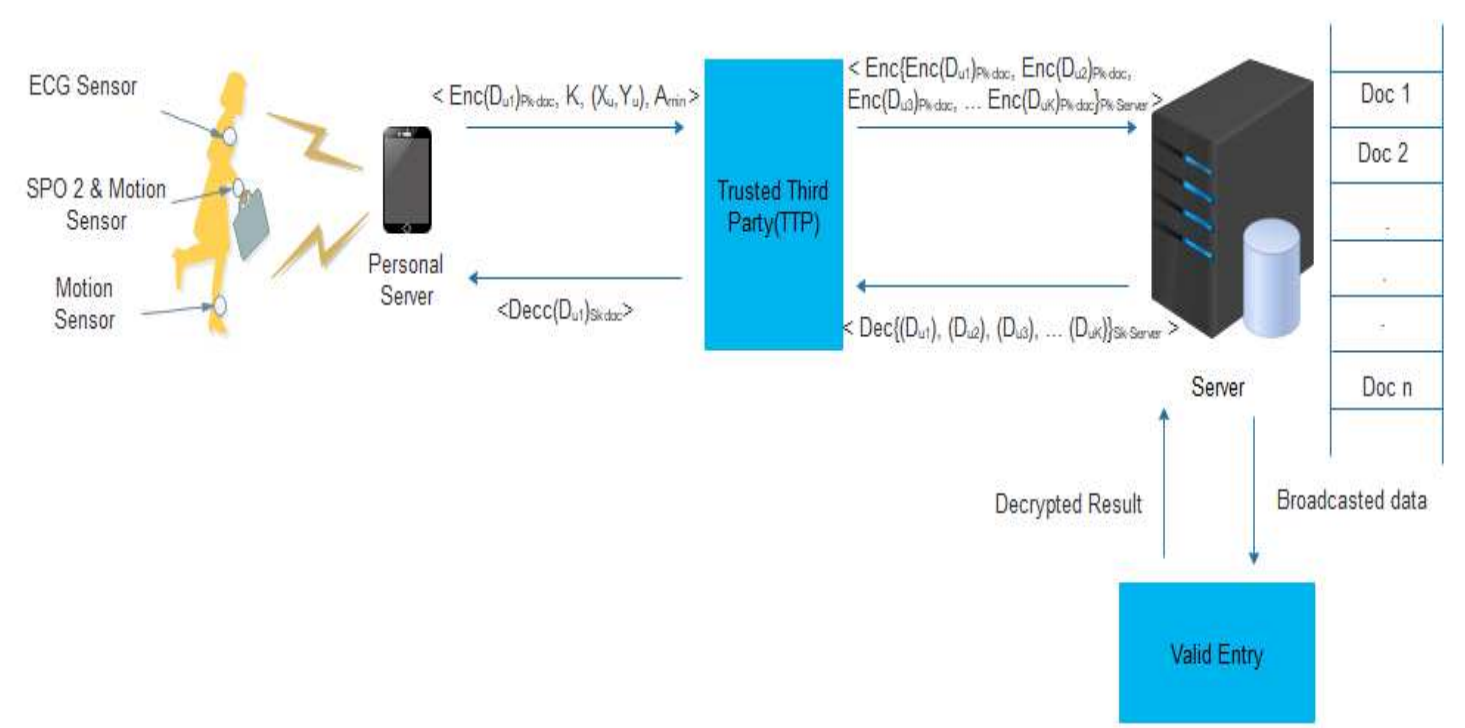

Figure 3: Proposed System Model of ECC.

\section{PROPOSED MODEL}

Our proposed system model solves the location privacy issue. Fig. 3 shows the proposed system model, sensors are placed on a patient's body and routinely gather data for example, pulse, blood pressure, internal heat level, etc. and all data is gathered into PDA (Personal Digital Assistant). If PDA records any fluctuation in the patient's body, it sends query ' $Q$ ' to the server that query indicating that patient's medical condition is serious. All collected data is encrypted using the Elliptic Curve Cryptography technique. In these circumstances, it is imperative to shield the current location information from the noxious client. PDA sends its original location coordinates along with K-anonymity parameter, patient data, K-anonymity minimum range to the trusted third party (TTP) in the encrypted form. TTP creates multiple dummy users with the Kanonymity parameter value to protect the current location of user and personal details (medical history and user identification). TTP encrypts the multiple user data with the server's public key and all the data (original user data and multiple user data) is sent to the server end. Server checks the key string in the list available on the server, user query ' $Q$ ' broadcasts to neighbor doctors. The key entry list verifies the valid entry of doctor's, with secret key server decrypts all user's data. All decrypted data is sent to the TTP. TTP verifies the original user, and encrypt that data with the public key 
of the sender and sent response back to the sender. Sender decrypts the data with private key of sender and gets the original message. If query is not matched then this query is terminated and user resends the 'Q' query.

\section{Elliptic Curve Cryptography}

Elliptic Curve Cryptography (ECC)is a public key cryptography technique, invented by two independent mathematic professors Neal Koblitz and Victor S. Miller in 1985. ECC was used widely in 2004 and 2005. ECC is appropriate for digital signatures, key arrangement, pseudo-random generators, and other functions. In Fig.4 ECC is used for encryption of the plain text into the ciphertext.

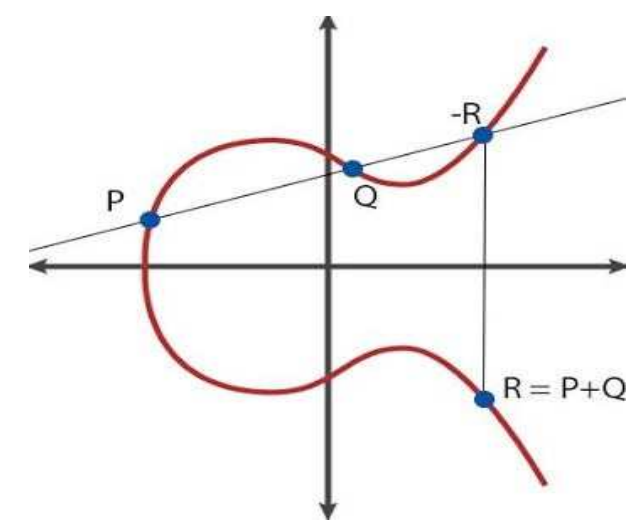

Figure 4: Elliptic Curve Cryptography [4].

The sender uses a key pair for encryption of the original message with the symmetric key encryption algorithm. ECC curve is a plane curve over a finite field that consists of the point that justifies the equation. The following formula is used for generating an ECC curve: $y^{2}=x^{3}+a x+b$.

In this it is easy to compute the one direction on the curve yet difficult to compute the opposite direction. If ' $t$ ' is given then finding the inverse becomes easy. The infinity point is showing the identity of the element. This technique preserves the location privacy of the user. User encrypts the message using the public key. Encrypted message and query ' $Q$ ' send into the database and a valid user decrypts the message through the private key. Elliptic Curve Cryptography is a technique used to preserve the location coordinates. Encryption and decryption are done with the public key and private key pair. This technique defined the ' $P$ ' represent the plain text and ' $C$ ' represents the ciphertext ' $E$ ' $\&$ ' $C$ ' represents the encrypted message. Table: 2 represent in no. of bits in RSA and ECC. It shows ECC use lesser number of bits than RSA. In this $(X, Y)$ is the location coordinates at the initial point. In this Elliptic Curve Cryptography, the standard formula is used for encryption and decryption. It is an asymmetric cryptography technique, in this encryption is performed with $E N C$ $P_{k}$ and decryption is with $D E C D_{K}$. The $E N C P_{k}$ takes a plain text and encrypts it using the public key. User medical data and query 'Q' sent to the database into the encrypted format. Query 'Q' match with the database with the list available on the server only valid user can decrypt the message using private key $D E C D_{K}$ of doctors.

\section{Considerations and Assumptions}

- The location services are active the mobile device of the patient.

- The mobile device uses the global positing system for finding the shortest way to the hospital.

- The patient is registering with the hospital. 
- Data is sent in the encrypted form.

- Only authorized person can decrypt the data.

- The proposed technique is providing a smaller key size and faster performance than the previous one.

- Location services data is used by the doctor, hospital staff, and ambulance.

\section{Steps for Communication in Proposed Model}

Communications steps of a proposed model for preserving the location privacy of the user. Let ' $p$ ' describes the point on a curve. If a line draws on the curve it will intersect only three points. ' $G$ ' generator point on the curve whose order is greater than $(\mathrm{n}>\mathrm{G})$. ' $S$ ' private key for decryption. ' $P$ ' public key used for encrypting the user data. ' $K$ ' anonymity parameter for creating the multiple dummy users. $X, Y$ location coordinates.

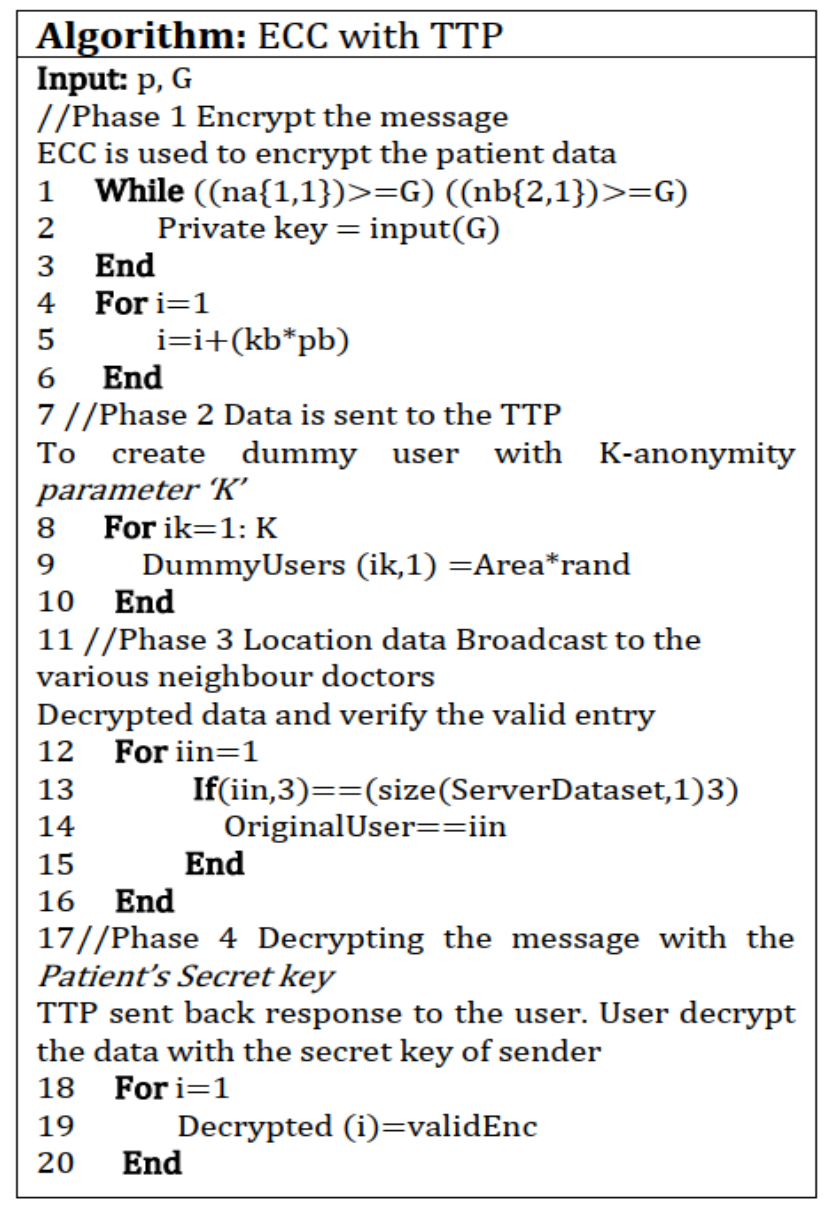

\section{Phase 1: Initial Stage User Encrypts The Message}

In phase 1 firstly, draw a curve using the random integer between 1 to $n-1$. The Addition of two points find third point $X+Y=Z$ reflects that the point across symmetric $\mathrm{x}$-axis. User sends its medical data and personal identity to the TTP in the encrypted format using ECC encryption technique. 


\section{Phase 2: Data is Sent to the Trusted Third Party (TTP)}

In phase 2, encrypted data is sent to the trusted third party. TTP created the multiple users with respect to ' $K$ ' value < $\operatorname{Enc}\left\{\operatorname{Enc}\left(D_{\mathrm{u} 1}\right)_{\mathrm{Pk}-\mathrm{doc}}, \operatorname{Enc}\left(\mathrm{D}_{\mathrm{u} 2}\right)_{\mathrm{Pk}-\mathrm{doc}}, \operatorname{Enc}\left(\mathrm{D}_{\mathrm{u} 3}\right)_{\mathrm{Pk}-\mathrm{doc}}, \ldots \operatorname{Enc}\left(\mathrm{D}_{\mathrm{uK}}\right)_{\mathrm{Pk}-\text { doc }}\right\}_{\text {Pk-Server }}>$ in the anonymity region. TTP creates the multiple dummy users, data is sent to the server.

\section{Phase 3: Location Data Broadcast to the Various Neighbor Doctors}

In this phase server checks key string in the list available on the server. Broadcast the data to neighbor doctor's and verify the valid entry. Server then decrypts the message with the private key of server, decrypted data is sent to the Trusted Third Party.

\section{Phase 4: Decrypting the Message with the Patient's Secret Key}

In phase 4, all the decrypted is sent to TTP. TTP verifies the original user, and encrypt that data with the public key of the sender and sent response back to the sender. Sender decrypts the data with private key of sender and gets the original message.

\section{EXPERIMENTAL RESULTS}

The execution and simulation scenario of the proposed model are carried out in the MATLAB. To evaluate the performance with various K- anonymity parameter, encryption and decryption process is evaluated. The time noted for encryption process, the consequences of the encryption are assessed and contrasted with the base of encryption time and size of message is encrypted. The outcome acquired from the encryption process with a current best answer for investigation. The size of the information record scrambled, and time required for the encryption by our proposed arrangement was estimated from the simulation process.

Encryption Time: It is determined in seconds. ECC encryption time is lesser than the RSA as shown in Fig. 5. ECC encryption time is faster also its key size small than another encryption technique. The outcome from this correlation is appeared in Table: 3 .

Decryption Time: Fig. 6 showing the ECC decryption is better than the RSA. ECC takes 0.0022437 RSA takes 0.31754 with the value of $\mathrm{K}=5$.

K-anonymity: All user data is sent to the TTP, it creates a multiple dummy user with the K-anonymity parameter value. It shows the impact on the encryption and decryption time and reduced the message size with the distinct values of $\mathrm{K}$-anonymity with ' $\mathrm{K}$ ' parameter.

Message size: Fig. 7 sums up the information sizes handled by various calculations, and shows our proposed calculation can deal with the message size that is reduced than RSA. ECC takes less memory to store the data. 
Encryption Time with the K-Anonymity Values

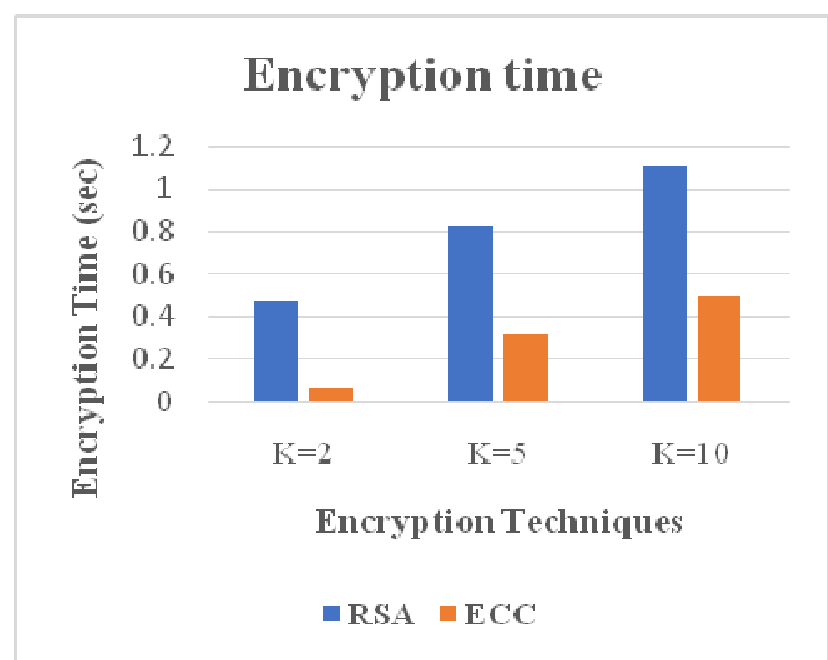

Figure 5: Encryption Time Compared with RSA.

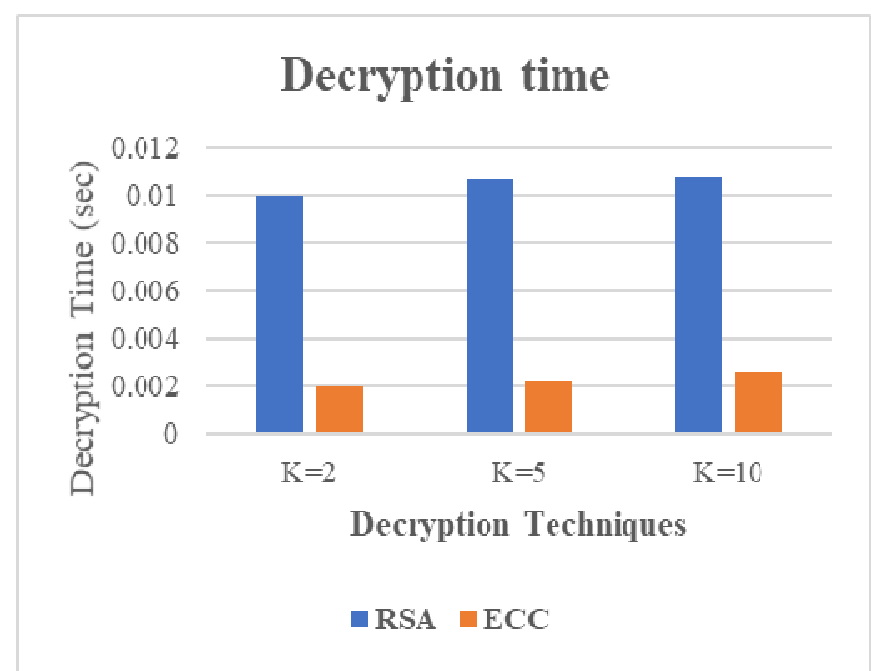

Figure 6: Decryption Time Compared with RSA.

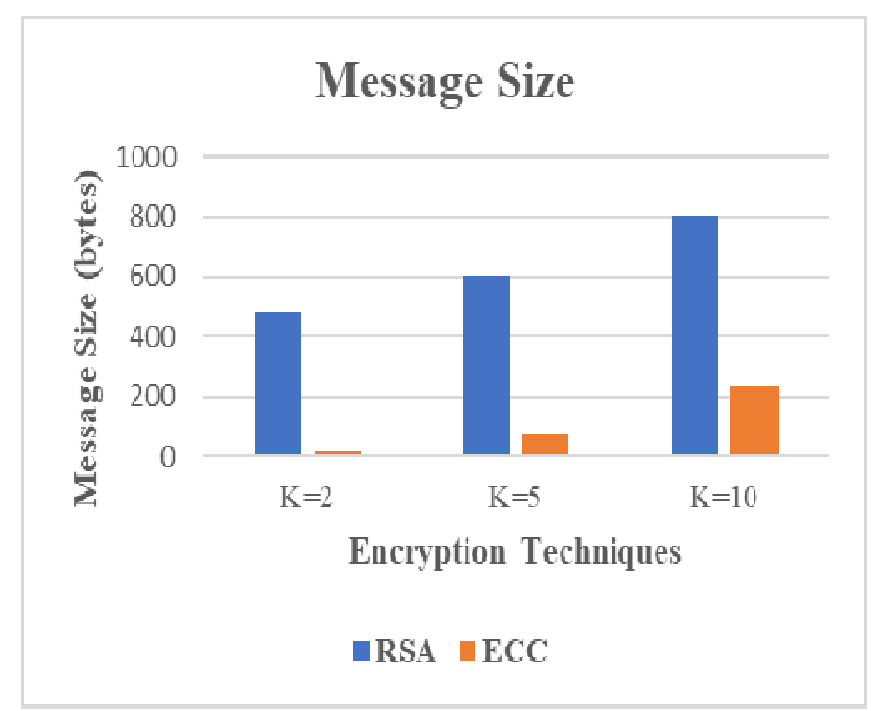

Figure 7: Message Size Compared with RSA. 
Table 3: Results Compared with the RSA and ECC

\begin{tabular}{|c|c|c|c|c|c|c|}
\hline K-anonymity & \multicolumn{3}{|c|}{ RSA } & \multicolumn{3}{c|}{ Proposed Solution } \\
\hline & $\begin{array}{c}\text { Encryption } \\
\text { time(sec) }\end{array}$ & $\begin{array}{c}\text { Decryption } \\
\text { time(sec) }\end{array}$ & $\begin{array}{c}\text { Message } \\
\text { size(bytes) }\end{array}$ & $\begin{array}{c}\text { Encryption } \\
\text { time(sec) }\end{array}$ & $\begin{array}{c}\text { Decryption } \\
\text { time(sec) }\end{array}$ & $\begin{array}{c}\text { Message } \\
\text { size(bytes) }\end{array}$ \\
\hline $\mathrm{K}=2$ & 0.46864 & 0.010011 & 480 & 0.067545 & 0.0019937 & 16 \\
\hline $\mathrm{K}=5$ & 0.81864 & 0.010661 & 603 & 0.31754 & 0.0022437 & 73 \\
\hline $\mathrm{K}=10$ & 1.1036 & 0.010776 & 803 & 0.49254 & 0.0026187 & 233 \\
\hline
\end{tabular}

This is on the grounds that the proposed encryption framework utilizes ECC calculation, which is fast and requires less vitality than RSA. Our proposed mode can offer high protected data transmission from the malicious user. It presents diverse reaction in Table: 3 on the message size, encryption and decryption time on both the encryption techniques but ECC is better than the previous techniques.

\section{CONCLUSIONS}

This paper proposes an ECC based location privacy mechanism to shield location privacy of the user. Our proposed technique good at shielding the patient details from the malicious user. Earlier in WBAN, to secure the patient data and use the RSA only to use the digital signature concept to make it safe from the denial. ECC contributes to the security and authentication of the user details. The proposed model uses Elliptic Curve Cryptography with the TTP safely transmit the data to the server through the communication medium. The performance of the ECC technique with the K-anonymity parameter shows better results than the previous one. It serves better security to the patient location and the personal details. The best thing about this model works on the emergency situation first and analyzed all the patient details before going to the near hospital. With the support of the ECC. It offers the better encryption time and generation of the key faster than the RSA.

\section{FUTURE SCOPE}

The proposed model is used to preserve the location privacy of the user. Our technique provides more security in lesser number of bits with faster generation of keys for secure communication. In both the cases this technique is work, to handle emergency patient and regular patients. In future, holds the promising field for introducing more security of combining software-defined networks with WBAN for more security of user data.

\section{Conflict of Interest}

The author declares that there is no conflict of interest regarding the publication of paper.

\section{REFERENCES}

1. Morales, L. V., Delgado-Ruiz, D., \& Rueda, S. J. (2019). Comprehensive Security for Body Area Networks: A Survey. International Journal of Network Security, 21(2), 342-354.

2. Hasan, K., Biswas, K., Ahmed, K., Nafi, N. S., \& Islam, M. S. (2019). A comprehensive review of wireless body area network. Journal of Network and Computer Applications, 143, 178-198

3. McGhin, T., Choo, K. K. R., Liu, C. Z., \& He, D. (2019). Blockchain in healthcare

4. applications: Research challenges and opportunities. Journal of Network and Computer Applications.

5. Konan, M., \& Wang, W. (2019). A Secure Mutual Batch Authentication Scheme for Patient Data Privacy Preserving in WBAN. Sensors, 19(7), 1608. 
6. Pramanik, P. K. D., Pareek, G., \& Nayyar, A. (2019). Security and Privacy in Remote Healthcare: Issues, Solutions, and Standards. In Telemedicine Technologies (pp. 201-225). Academic Press.

7. Wang, G., Lu, R., \& Guan, Y. L. (2019). Achieve privacy-preserving priority classification on patient health data in remote eHealthcare system. IEEE Access, 7, 33565-33576.

8. Zhen, Y., \& Liu, H. (2019). Distributed privacy protection strategy for MEC enhanced Wireless Body Area Networks. Digital Communications and Networks.

9. Gupta, R., \& Rao, U. P. (2019). VIC-PRO: Vicinity protection by concealing location coordinates using geometrical transformations in location based services. Wireless Personal Communications, 107(2), 1041-1059.

10. Sun, W., Cai, Z., Li, Y., Liu, F., Fang, S., \& Wang, G. (2018). Security and privacy in the medical internet of things: a review. Security and Communication Networks, 2018.

11. Gupta, R., \& Rao, U. P. (2017). A hybrid location privacy solution for mobile LBS. Mobile Information Systems, 2017.

12. Gupta, R., \& Rao, U. P. (2017). Achieving location privacy through CAST in location based services. Journal of Communications and Networks, 19(3), 239-249.

13. Gupta, R., \& Rao, U. P. (2017). An exploration to location based service and its privacy preserving techniques: a survey. Wireless Personal Communications, 96(2), 1973-2007.

14. Olakanmi, O. O. (2017). Lightweight security and privacy scheme for wireless body area network in e-health system. Int. J. of Information Security Science, 6(3), 26-38.

15. Khan, F. A., Ali, A., Abbas, H., \& Haldar, N. A. H. (2014). A cloud-based healthcare framework for security and patients' data privacy using wireless body area networks. Procedia Computer Science, 34, $511-517$.

16. Lee, Y. S., Alasaarela, E., \& Lee, H. J. (2014). An efficient encryption scheme using elliptic curve cryptography (ECC) with symmetric algorithm for healthcare system. International journal of security and its applications, 8(3), 63-70.

17. Sinha, R., Srivastava, H. K., \& Gupta, S. (2013). Performance based comparison study of RSA and elliptic curve cryptography. International Journal of Scientific \& Engineering Research, 4(5), 720-725.

18. Mallikarjuna D S, K. N. Raja Rao, Abha Agarwal \& Debajit Dutta, "Dual-Cell HSDPA Operation and its Performance Evaluation “, International Journal of Electronics and Communication Engineering (IJECE), Vol. 3, Issue 4, pp. 11-20

19. B. Muniswamy \& N. Geethanjali, "A Review on Network Coding and its Applications in Wired and Wireless Networks “, IMPACT: International Journal of Research in Engineering \& Technology (IMPACT: IJRET), Vol. 2, Issue 5, pp. 179-186

20. Samer I. Mohamed \& Amr Abdelnabi, "Agent-Based Convolution and Reinforcement Learning “, BEST: International Journal of Management Information Technology and Engineering (BEST: IJMITE), Vol. 5, Issue 12, pp. 17-28

21. Shivani Shankar, "Internet of Things: An Overview “, International Journal of Computer Science and Engineering (IJCSE), Vol. 5, Issue 4, pp. 23-30. 